\title{
Geometric Characterization of Series-Parallel Variable Resistor Networks*
}

\author{
Randal E. Bryant \\ J. D. Tygar \\ School of Computer Science \\ Carnegie Mellon University \\ Pittsburgh, PA 15213 USA
}

\author{
Lawrence P. Huang \\ IBM Corporation \\ 11400 Burnet Road \\ Austin, TX 78758
}

\begin{abstract}
The range of operating conditions for a seriesparallel network of variable linear resistors, voltage sources, and current sources can be represented as a convex polygon in a Thevenin or Norton half plane. For a network with $k$ variable elements, these polygons have at most $2 k$ vertices. By introducing a class of infinite points, we can also represent circuits with potentially infinite Thevenin resistance or Norton conductance.
\end{abstract}

\section{Introduction}

In analyzing a circuit under a range of operating conditions or parametric variations, three approaches are commonly followed. First, one can characterize the nominal behavior and express the effect of variations as sensitivities [2,3]. Such an approach is appropriate only when the variations are small. Second, one can employ Monte Carlo methods to statistically characterize the effects of variations. Finally, one can develop bounding techniques that succinctly characterize the potential range of behaviors [8]. Bounding approaches have the advantage that they capture the full range of behaviors with a single computation, and that they do not overlook any extreme, although statistically improbable, cases.

This paper considers methods to bound the range of behaviors of variable resistor networks. This problem arises when modeling MOS circuits by linear switch-level simulation [6]. In this model, transistors are modeled as switched, linear resistors, while node voltages are approximated by logic values $\{0,1, X\}$, where $X$ indicates an unknown or potentially nondigital voltage. When a transistor gate node has value $X$, the transistor is assumed to have an arbitrary resistance greater than or equal to its value when fully on. The simulator must then compute the ranges of possible steady state voltages on the nodes for all possible variations of the resistances to determine the new node states.

Most linear switch-level simulators use simplistic methods to compute the possible voltage ranges $[1,6]$. At times they

\footnotetext{
* This research was supported by the Defense Advanced Research Project Agency, ARPA Order 4976, by the National Science Foundation, PYI Grant CCR-8858087, and by the Semiconductor Research Corporation under Contract 91-DC-068
}

can produce results that are overly pessimistic, computing a larger range than is actually achievable, while at other times they produce results that are overly optimistic, computing a smaller range. In fact, existing programs can even fail to compute the correct result for fixed resistance networks.

In earlier work, we have shown that computing the precise range of voltages in an arbitrary network of variable, linear resistors is NP-complete [5]. Thus, it is unlikely that an efficient algorithm for this task exists. Instead, we must look for algorithms that either work under restricted conditions, or for efficient algorithms that at times err on the side of pessimism. This paper describes an efficient method for computing exact bounds on the operating conditions of series-parallel networks. The method handles networks of independent, variable linear elements: resistors, voltage sources, and current sources. Arbitrary, nonnegative resistance values are allowed, including infinite ones. The method derives exact results for any physically realizable series-parallel network. In particular, it fails only under conditions where two voltage sources of potentially differing voltage are connected in parallel or where two current sources of potentially differing current are connected in series.

\section{Summary of Method}

Our method operates by taking a geometric view of the set of possible network operating points. The possible Thevenin or Norton equivalent circuits for the network are viewed as points in a half plane. Thevenin equivalents having finite resistance are represented by points of the form $\langle R, V\rangle$, while Norton equivalents having finite conductance are represented by points of the form $\langle G, I\rangle$. We also introduce a class of infinite "Omega" points to represent infinite resistances and conductances. That is, the Thevenin equivalent of a current source is given by Omega point $\langle\langle I\rangle\rangle$, while the Norton equivalent of a voltage source is given by Omega point $\langle\langle V\rangle\rangle$. We extend conventional Euclidean geometry to include Omega points in a straightforward way.

Our main result is to show that the Thevenin or Norton equivalent of a series-parallel network containing $k$ variable elements can be represented as a convex polygon of degree (i.e., number of vertices) less than or equal to $2 k$. Furthermore, if the network contains a total of $n$ elements, this polygon can be com- 
Thevenin Representation

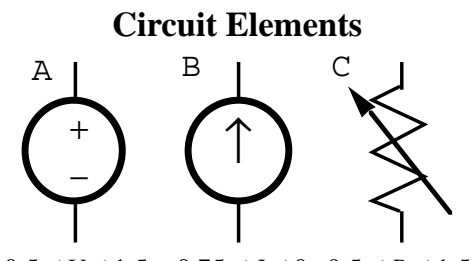

$0.5 \leq V \leq 1.5 \quad-0.75 \leq I \leq 0 \quad 0.5 \leq R \leq 1.5$

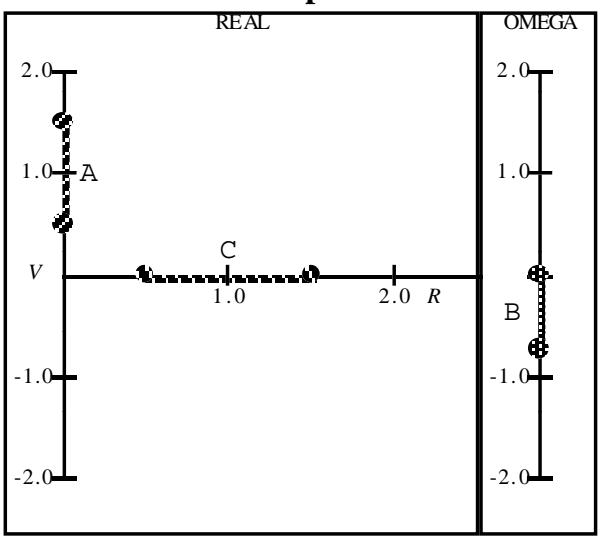

Norton Representation

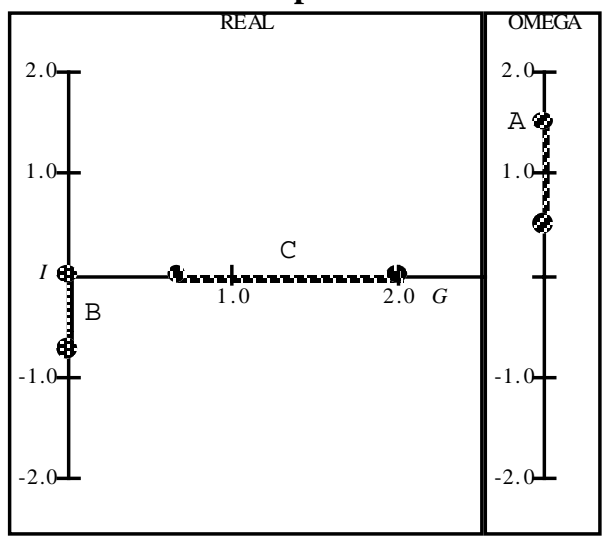

Figure 1: Variable Circuit Elements and their Representations
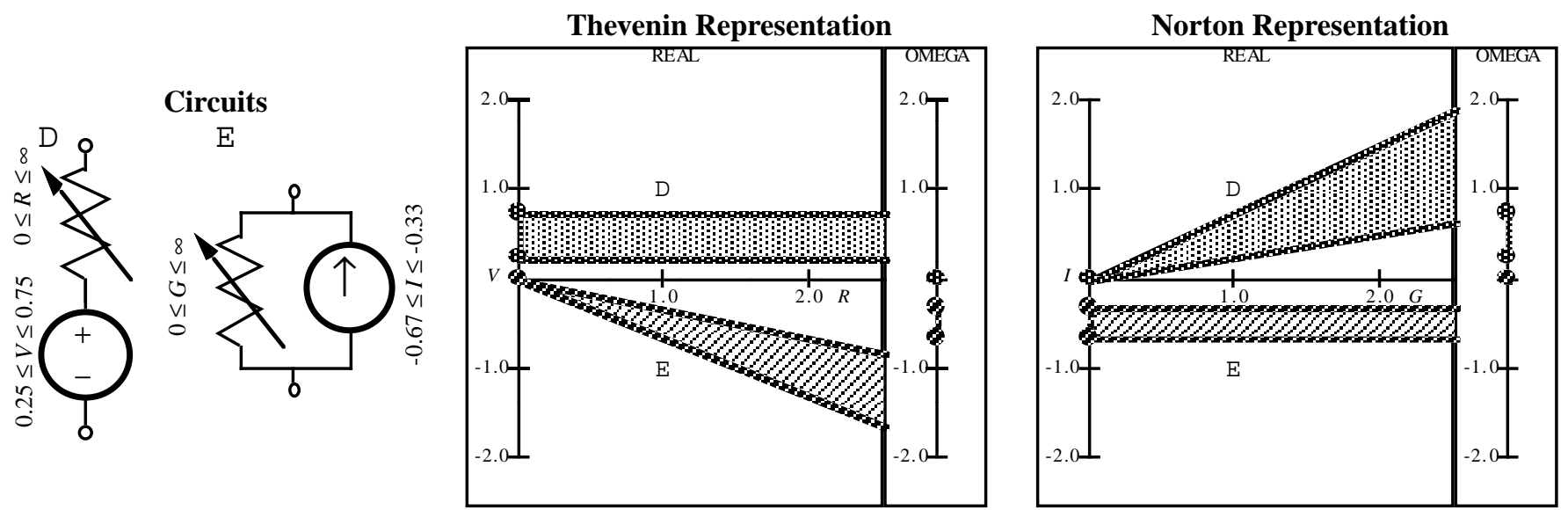

Figure 2: Two Element Circuits and their Representations

puted in time $O(n k)$. Given such a polygon, one can easily determine the ranges of possible steady state voltages, currents, resistances, or conductances.

\section{Thevenin and Norton Representations of Circuits}

We will refer to the two coordinate systems for representing a circuit as the Thevenin and Norton half planes. Figure 1 illustrates the representations of the allowed circuit elements. Observe in this figure that the $X$ axis (resistance in the Thevenin plane and conductance in the Norton) extends indefinitely far to the right. We show the set of Omega points along a separately axis to the right of all real points; conceptually these points correspond to infinite values of resistance or conductance. Note that the vertical scale for Omega points will generally differ from that for real points. A voltage source varying over the voltage interval $\left[V_{\min }, V_{\max }\right]$ (circuit $\mathrm{A}$ ) is represented in the Thevenin plane as a line segment along the $Y$ axis having end points $\left\langle 0, V_{\min }\right\rangle$ and $\left\langle 0, V_{\max }\right\rangle$ indicating that its Thevenin resistance is 0 . The same source is represented in the Norton plane as a line segment along the Omega axis having endpoints $\left\langle\left\langle V_{\min }\right\rangle\right\rangle$ and $\left\langle\left\langle V_{\max }\right\rangle\right\rangle$, indicating that it has infinite Norton conductance. The representations of a current source (circuit B) are the duals of those for a voltage source-either a segment along the Omega axis in the Thevenin plane or a segment along the
$Y$ axis in the Norton plane.

A resistor varying over the (finite, nonzero) resistance interval $\left[R_{\min }, R_{\max }\right]$ (circuit $\mathrm{C}$ ) is represented in both Thevenin and Norton planes as a horizontal line segment along the $X$ axis. In the Thevenin plane this segment has endpoints $\left\langle R_{\min }, 0\right\rangle$ and $\left\langle R_{\max }, 0\right\rangle$, while in the Norton plane it has endpoints $\left\langle 1 / R_{\max }, 0\right\rangle$ and $\left\langle 1 / R_{\min }, 0\right\rangle$. For a resistor with $R_{\max }=\infty$ (i.e., an open circuit), the Thevenin representation would still be a segment, but the right hand endpoint would be the Omega point $\langle\langle 0\rangle\rangle$ and the segment would contain all real points $\langle R, 0\rangle$ for $R$ greater or equal to $R_{\min }$. Similarly, for a resistor with $R_{\text {min }}=0$ (i.e., a perfect conductor), the Norton representation would be a segment with right hand endpoint $\langle\langle 0\rangle\rangle$.

Figure 2 illustrates the Thevenin and Norton representations of circuits consisting of a single variable source and a single variable resistor varying over all possible resistances (i.e., from 0 to $\infty$ ). Observe that the Thevenin representation of voltage source ranging over the interval $\left[V_{\min }, V_{\max }\right]$ plus series resistor (circuit $D$ ) is a rectangle- the source voltage and the Thevenin resistance are independent, plus the Omega point $\langle\langle 0\rangle\rangle$ indicating that when the resistance becomes infinite, the Thevenin representation is that of an open circuit. The Norton representation of this circuit is more subtle. When the conductance is infinite, the circuit behaves as a variable voltage source, 

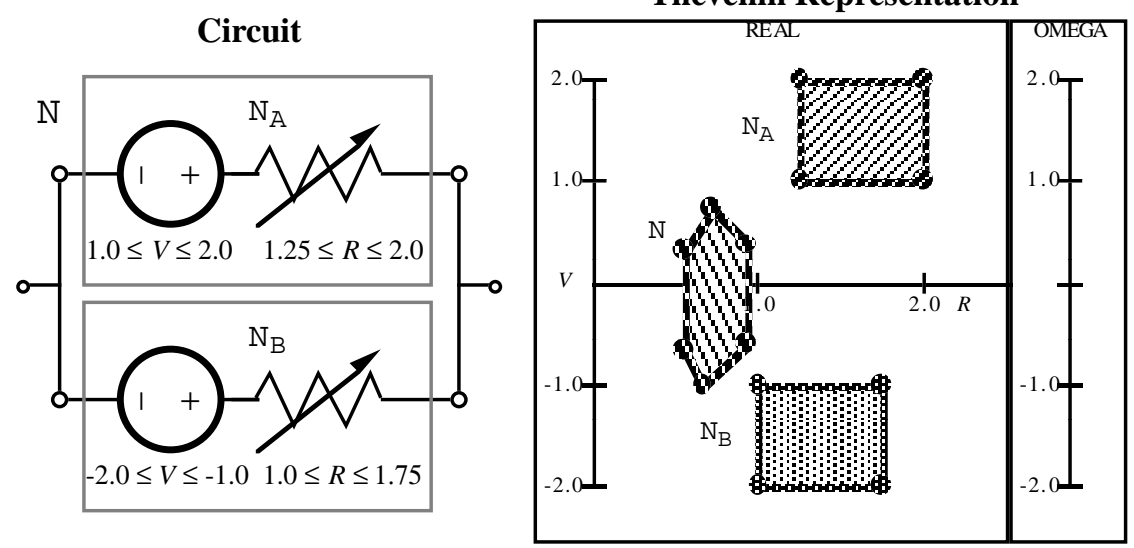

Norton Representation

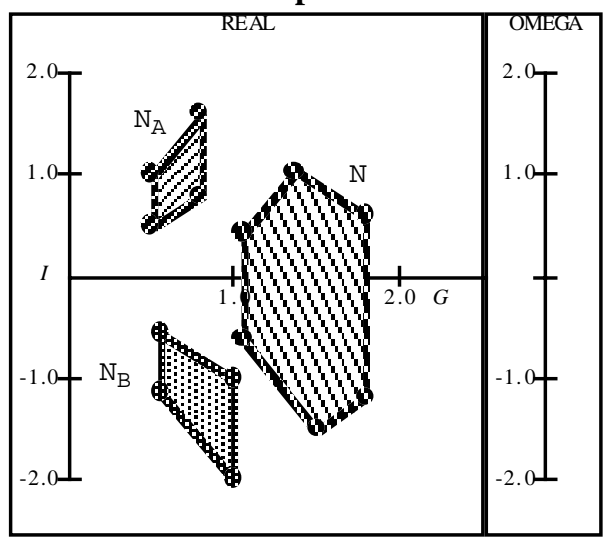

Figure 3: Parallel Combination of Networks

represented by a segment along the Omega axis. As the conductance is decreased, the Norton conductance becomes finite with the current range bounded by two lines with slopes $V_{\min }$ and $V_{\max }$. As the conductance approaches zero, the Norton representation is that of an open circuit, i.e., the real point $\langle 0,0\rangle$. The dual case occurs for a variable current source in parallel with a variable resistance (circuit $E$ ).

Figure 3 illustrates the effect of combining several smaller networks of variable elements. Both networks $\mathrm{N}_{\mathrm{A}}$ and $\mathrm{N}_{\mathrm{B}}$ consist of voltage sources and series resistances. Hence their Thevenin representations are rectangular. However, when these networks are combined in parallel to form network $\mathrm{N}$ the overall range of possible Thevenin equivalents is given by a hexagon. A method for deriving this polygon will be described in the next section. It involves transforming the two rectangles in the Thevenin plane into the trapezoids shown in the Norton plane. These trapezoids are then "summed" giving a hexagon describing the range of Norton equivalents for network $\mathrm{N}$. This polygon is then transformed back to the Thevenin plane.

\section{Computational Method}

Each network element is represented as either a point (for a fixed element) or a line segment (for a variable element). Following the series-parallel structure of the network we construct polygonal representations of each subnetwork, converting to a Norton form for parallel connections and to a Thevenin form for series connections. As illustrated in Figure 4, a polygon is represented by its upper and lower contours, consisting of the set of vertices along the upper or lower boundary of the polygon. As this example illustrates, the final point in a contour may be an Omega point $\langle\langle m\rangle\rangle(m=-0.25$ in these cases $)$. Such a point defines a polygon edge extending from the preceding point of the contour infinitely to the right and having slope $\langle\langle m\rangle\rangle$.

We define a transform operation $\tau$ that converts between the Thevenin and Norton representations of a subnetwork as follows:

1. For real point $\langle x, y\rangle$ with $x>0: \tau(\langle x, y\rangle)=$ $\langle 1 / x, y / x\rangle$.

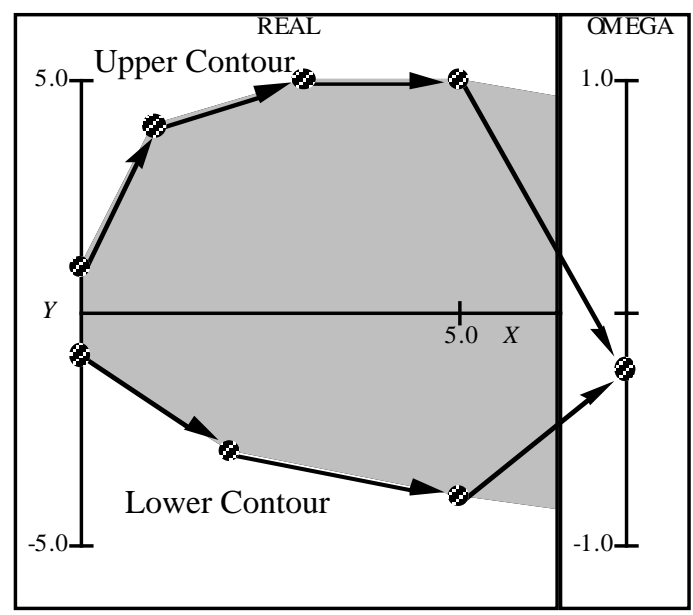

Figure 4: Contour Representation of a Polygon

2. For real point $\langle 0, y\rangle: \tau(\langle 0, y\rangle)=\langle\langle y\rangle\rangle$.

3. For omega point $\langle\langle m\rangle\rangle: \tau(\langle\langle m\rangle\rangle)=\langle 0, m\rangle$.

This operator has the properties that it preserves convexity and serves as its own inverse. In fact, the transform of a convex polygon is itself a convex polygon having as vertices the transformed vertices of the original polygon. Furthermore, since the transform preserves vertical orderings of points, the transformed vertices of the upper (respectively, lower) contour become the upper (resp., lower) contour of the transformed polygon. The left to right ordering of the points in the two contours is reversed, however.

We combine polygons by pointwise addition, yielding either the Norton representation of two subnetworks connected in parallel or the Thevenin representation of two subnetworks connected in series. Addition of a real point with an Omega point yields the Omega point, corresponding to the property that the parallel combination of a voltage source with a circuit having finite conductance yields the voltage source, and similarly for the series combination of a current source with a circuit having finite resistance. Addition of two identical Omega points yields the same point. This corresponds to the 

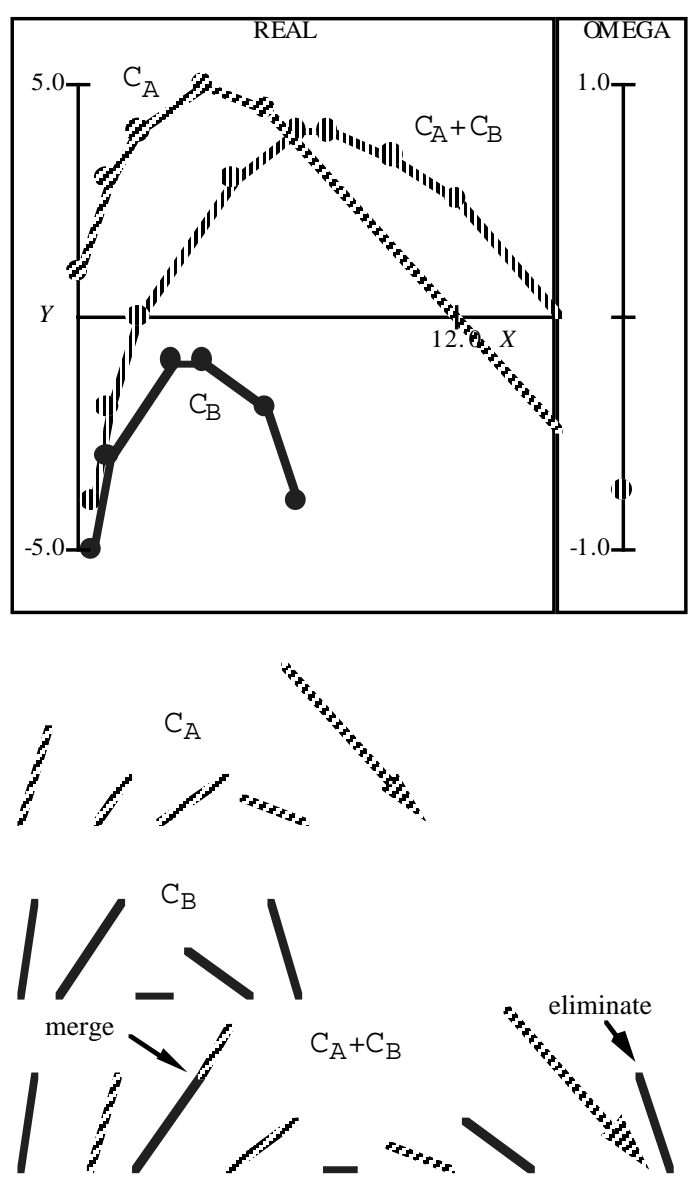

Figure 5: Contour Addition by Segment Merging

case where matching voltage (resp., current) sources are connected in parallel (resp., series). On the other hand, the sum of two distinct Omega points is undefined, corresponding to one of the error conditions described earlier.

Given the upper and lower contours of two polygons, one can easily be compute the upper and lower contours of their sum. This process is illustrated in Figure 5 for two upper contours. The ordered list of vertices in a contour define an ordered set of line segments, as shown in the lower part of the figure, each having the slope and length of an edge of the polygon. For an upper contour, the slopes of the segments will be in descreasing order. As this figure illustrates, the final segment of a contour may include an Omega point. To sum two contours $C_{A}$ and $\mathrm{C}_{\mathrm{B}}$, we start by merging the two segment lists into a single list in descending slope order. Where the two lists contain line segments of matching slope, we combine these into single segments as shown by the case labeled "merge" in the figure. We also eliminate any segments to the right of one containing an Omega point. The resulting list then becomes the set of segments in the sum. The upper contour $\mathrm{C}_{A}+\mathrm{C}_{B}$ has as leftmost vertex the sum of the leftmost vertices of $C_{A}$ and $C_{B}$. The remaining vertices are computed by adding the offset from the preceding vertex defined by the next segment in the list. A similar process is used for summing lower contours, except that the segment lists are in ascending slope order.

It can be shown that the sum of two convex polygons of degrees $k_{1}$ and $k_{2}$ is a convex polygon of degree less than or equal to $k_{1}+k_{2}$. Computing this sum has complexity $O\left(k_{1}+\right.$ $\left.k_{2}\right)$. Thus, for a network of $k$ variable elements, the Thevenin and Norton polygons will have degree at most $2 k$.

\section{Conclusions}

We have analyzed a number of university and industrial MOS circuit designs to determine how often a series-parallel network solution technique could be employed [4]. Even assuming worst case conditions where all of the transistors are potentially conducting, we determined that over $90 \%$ of the node voltages could be computed by this means. Under more realistic operating conditions, we would expect the technique to be applicable for many of the remaining $10 \%$.

For (two-port) networks that are not series-parallel, it can be shown by network tearing [7] that the range of Thevenin and Norton equivalents are also be given as polygons. However, these polygons may be concave and it appears they may have degree exponential in the number of variable elements.

\section{References}

[1] C.-Y. Chu, "Improved Models for Switch-Level Simulation," PhD Thesis, Stanford Dept. of EE, 1988.

[2] C. A. Desoer and E. S. Kuh, Basic Circuit Theory, McGrawHill, 1969.

[3] S. W. Director and R. A. Rohrer, "The Generalized Adjoint Network and Network Sensitivities," IEEE Trans. on Circuit Theory, 16(3):318-323, Aug., 1969.

[4] L. P. Huang, "Modeling Uncertainty in Linear Switch-Level Simulation," PhD Thesis, CMU Dept. of ECE, 1991.

[5] L. P. Huang, and R. E. Bryant, "Intractability and SwitchLevel Simulation," in press, 1993.

[6] C. J. Terman, "Simulation Tools for Digital LSI Design," PhD Thesis, MIT Dept. of EECS, 1983.

[7] R. A. Rohrer, "Circuit Partitioning Simplified," IEEE Trans. CAS, CAS-35(1), Jan., 1988.

[8] C. A. Zukowski, The Bounding Approach to VLSI Circuit Simulation, Kluwer, 1986. 